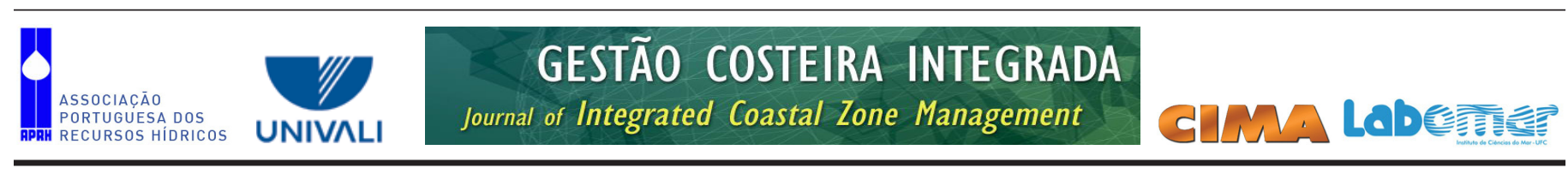

http://www.aprh.pt/rgci/pdf/rgci-382_Moretz-Sohn.pdf | DOI:10.5894/rgci382

\title{
Pescadores artesanais e a implementação de áreas marinhas protegidas: Estudo de caso no nordeste do Brasil *
}

\author{
Artisanal fishermen and implementation of marine protected areas: \\ A case study of northeastern Brazil
}

\author{
Clarissa Dantas Moretz-Sohn ${ }^{1}$, Thaysa Portela Carvalho ${ }^{1}$, Francisco Jailton Nogueira Silva Filho ${ }^{1}$, \\ Francisco Gleidson da Costa Gastão ${ }^{1}$, Danielle Sequeira Garcez ${ }^{1}$, Marcelo de Oliveira Soares 1,@
}

\section{RESUMO}

$\mathrm{Na}$ última década, a criação de áreas marinhas protegidas nas zonas costeiras aumentou consideravelmente. No entanto, é comum em diversos países a inexistência ou pouca eficácia no processo de planejamento participativo na criação destas unidades de conservação, o que pode ocasionar diversos conflitos sócio-ambientais, sobretudo com as comunidades tradicionais. Este estudo tem por objetivo analisar como a percepção ambiental de pescadores artesanais pode contribuir para análise e criação de uma área marinha protegida (AMP) no Nordeste do Brasil (Icapuí, Ceará), em uma região de reconhecido conflito entre os modos de pesca. Esta região é conhecida principalmente pela pesca da lagosta (Panulirus spp.), recurso que apresentou significativo declínio nos últimos 50 anos devido à capturas ilegais. A criação de uma AMP foi analisada sob a ótica dos pescadores artesanais, enfocando um diagnóstico ambiental participativo e as possibilidades de uso sustentável da área. Para atingir o objetivo proposto, o perfil sócio-econômico e os principais recursos pesqueiros foram analisados segundo questionários semi-estruturados aplicados, e entrevistas. Foram também analisadas a percepção ambiental referente à área marinha protegida proposta e a categoria de uso mais adequada, segundo os partícipes. Os resultados da pesquisa comprovaram que a população depende diretamente da atividade pesqueira, e que os pescadores possuem ampla percepção sobre as relaçóes ecossistêmicas na região. Além disso, todos os moradores da área estudada reconheceram a importância e necessidade da implantação de uma Área Marinha Protegida visando, principalmente, o ordenamento pesqueiro. Essa necessidade náo se aplica somente à área em questão e sim a uma abrangência de praias maior, cujos territórios marinhos se estendem além dos limites correspondentes em terra. Pretende-se que o diagnóstico participativo apresentado, baseado na percepção ambiental de pescadores artesanais residentes, sirva de base para discussão dos problemas relacionados ao gerenciamento das áreas marinhas protegidas, os quais devem compatibilizar a exploração dos recursos pesqueiros com a conservaçáo dos bens/serviços ambientais.

Palavras-Chave: Gestão Ambiental, Pesca Artesanal, Sustentabilidade.

\section{ABSTRACT}

Over the past decade, the establishment of Marine Protected Areas (MPAs) in coastal zones has increased considerably. However, it is common in many countries the absence or ineffectiveness of the process of participatory planning in the creation of these protected areas, which can cause several socio-environmental conflicts, especially with traditional communities. This paper aims to examine the use of environmental perception for analysis and creation of an MPA in northeastern Brazil (Icapui, Ceará), a region recognized by the conflict between modes of its coastal fisheries. This region is known mainly for its lobster (Panulirus spp.) fishery, which has had a significant decline in the last 50 years due to illegal and

@ - Autor para correspondência: marcelosoares@ufc.br

1 - Instituto de Ciências do Mar (LABOMAR), Universidade Federal do Ceará, CEP 60165-081, Fortaleza, CE, Brasil.

* Submission: November 8, 2012; Evaluation: December 17, 2012; Reception of revised manuscript: May 31, 2013; Accepted: June 13, 2013; Available on-line: June 17, 2013 
excessive fishing. It is relevant to mention that the lobster, more than a fishing resource of high economic value, is a symbol of artisanal fisheries of the region. The creation of an MPA was analyzed from the perspective of the local fishermen, focusing on a participatory environmental diagnosis and possibilities for sustainable use of the area. To achieve this purpose, the socio-economic profile and the main fishing resources were analyzed using semi-structured questionnaires and interviews. We also analyzed the environmental perception regarding the proposed marine protected area and the most appropriate category of use, according to participants. The results showed that the population of this marine area depends directly on fishing activity, and that fishermen have a good perception of the ecosystem relations and resources in the region. In addition, all residents recognize the importance and need for the establishment of a Marine Protected Area, like community-based bottom-up MPA. An MPA would be a way to protect natural resources and encourage the adoption of conservation measures around the coast of Ceara. It is intended that the model of environmental perception and participatory diagnosis with traditional fishermen serve as an example for discussion of problems related to management of marine protected areas, which must reconcile the exploitation of fishing resources and the conservation of the property lenvironmental services.

Keywords: Environmental Management, Artisanal Fishery, Sustainability.

\section{INTRODUÇÃO}

A pesca artesanal agrega quase $90 \%$ do total de pescadores no mundo, representando um contingente de aproximadamente 40 milhóes de pessoas empregadas diretamente neste setor. Assim, é clara a importância econômica, sócio-ambiental e cultural que esta modalidade de atividade pesqueira ostenta (Begossi, 2004; Batista et al. 2011).

Esforços voltados para o desenvolvimento sustentável dos oceanos e dos ambientes costeiros resultaram na criação de áreas marinhas protegidas (AMPs) como um dos principais instrumentos do planejamento ambiental visando a conservação dos recursos ambientais, inclusive dos recursos pesqueiros em áreas de pesca artesanal (Gerhardinger et al., 2009; Ângulo-Valdes \& Hatcher, 2010; Suuronen et al., 2010; Ban et al., 2011). Reconhecida esta importância, nas últimas décadas, os ambientes costeiros e oceânicos, por meio de inúmeros dispositivos legais, passaram a ser protegidos em relação a seus serviços ecológicos e valores culturais (Agardy, 1994; Kelleher, 1999; Kenchington, 2010; Ransom \& Mangi, 2010). Um tipo de instrumento técnico e legal importantíssimo é a criação de AMP.

A AMP abrange diferentes tipos de proteção dependendo de seus objetivos conservacionistas, econômicos e sociais, e engloba desde áreas pequenas destinadas a proteger determinadas espécies em perigo de extinção, um hábitat, ou grandes áreas com variados ecossistemas (Jamieson \& Levings, 2001; Game et al., 2009; Read et al., 2011). Sáo objetivos da AMP garantir processos energéticos vitais como a manutençáo de teias alimentares, protegendo tanto a biodiversidade quanto a produtividade, e auxiliar na manutenção dos estoques pesqueiros, através da proteção às áreas de berçários e da exportação de recursos (peixes, crustáceos, moluscos) das zonas de extração restrita para o entorno (Kelleher, 1999). Estas AMPs podem ter (no caso brasileiro): 1) uma proteção integral, onde, por exemplo, tornam-se áreas de exclusão de pesca; ou 2) é permitido o uso sustentável, onde pode-se realizar pesca, porém com controle mais restrito de uso (por exemplo, somente a pesca artesanal).

Contudo, a implementação de AMPs tem causado conflitos entre os pescadores artesanais e outros atores sociais, e isto muitas vezes está atrelado ao fato de a AMP ter sido criada sem consulta à comunidade e sem a contextualização socioeconômica, cultural e ambiental da comunidade no plano gestor (Gerhardinger et al., 2009; Voyer et al., 2012). Através do levantamento do contexto socioeconômico e político da comunidade podemos analisar variáveis sociais que influenciarão o comportamento dos usuários do recurso e assim, organizar uma estratégia adequada de manejo. Além destes estudos, a percepção ambiental advinda da comunidade de pescadores pode ser uma variável importante no planejamento das novas unidades de conservação (Oliveira, 2002; Begossi, 2004; Cinner, 2007; Kenchington, 2010). A reduzida atenção ao contexto sócio-ambiental leva comumente a um fracasso com baixa efetividade do manejo em áreas marinhas protegidas (Cinner, 2007). Por outro lado, a identificação dos usuários, o conhecimento local e a inserção deles no plano gestor são essenciais para garantir a eficiência do manejo (Heck et al., 2012).

Além disso, agregando o conhecimento etnoecológico dos pescadores (CEP) (Berkes, 1993; Areizaga et al., 2012) ao conceito de gestáo baseada nos ecossistemas, onde os limites de proteção em sentido geográfico baseiam-se na medida dos movimentos de organismos e processos fisicamente ligados (Agardy, 2000; Scholz et al. 2004), gera-se uma sinergia entre o CEP e o conhecimento científico (Glaser et al., 2010). Assim, os planos gestores podem ser melhor desenvolvidos visando o manejo do ecossistema e não apenas um recurso individual.

A presença de populações tradicionais, suas atividades econômicas, seus conhecimentos da natureza são importantes para o planejamento das áreas marinhas protegidas. As diretrizes e as políticas para as unidades de conservação carecem de um debate com estas populações, como a inclusão dos praticantes de pesca artesanal, que são partícipes na vida local. Voyer et al. (2012) afirmam que devido às dificuldades associadas ao planejamento e ao manejo das áreas marinhas protegidas, normalmente espaços com conflitos de uso, é necessário reavaliar o modo como as consideraçốes econômicas e sociais são incorporadas nas atividades de planejamento, abordando que a consulta e análise da percepção das comunidades, antes da existência da AMP, é um caminho importante.

Apesar desta relevância e das Convençóes Internacionais (Conferência da Diversidade Biológica e da IUCN International Union of Conservation Nature) (Bogaert et al., 2009) abordarem a importância destes estudos, existem ainda poucas pesquisas no Brasil, conforme observado 
por Gerhardinger et al. (2009), enfocando como os pescadores artesanais podem auxiliar no processo de criação de áreas marinhas protegidas em ambientes costeiros tropicais, sobretudo em países em desenvolvimento de língua portuguesa. Assim, esta pesquisa visa analisar: 1) o contexto sócio-econômico e os recursos pesqueiros em uma comunidade de pescadores artesanais no Nordeste do Brasil (Praia de Picos, Icapuí, Ceará); 2) avaliar como a percepção ambiental e os conhecimentos etnoecológicos podem ser usados como ferramentas no planejamento da criação de uma AMP, enfocando a categoria desta unidade, o zoneamento ambiental e o grau de envolvimento e aceitação dos pescadores artesanais quanto à criação da mesma. Pressupóe-se que o planejamento participativo (desde o início), sobretudo com os pescadores, seja imprescíndivel para obter efetividade na criação e na gestão das AMPs.

Este levantamento é uma etapa inicial para idealização de estratégias de incentivo a diferentes níveis organizacionais das comunidades (familiar, comunitário ou de grupos de interesses comuns), para açóes que promovam o uso sustentável dos recursos pesqueiros em unidades de conservação. A área de estudo também possui interesse na análise em questão por apresentar forte conflito na pesca da lagosta; ocorrem métodos de pesca artesanais conflitantes com métodos reconhecidos como não-sustentáveis e ilegais em muitos países, como o uso de mergulhos com compressores para a prática de captura não seletiva.

\section{MATERIAL E MÉTODOS}

\section{1. Área de Estudo}

O município de Icapuí está localizado no Nordeste do Brasil, distando $206 \mathrm{~km}$ de Fortaleza (Capital do Estado do Ceará) (Figura 1) por via rodoviária, possuindo $64 \mathrm{~km}$ de praia, cerca de 16.000 habitantes e $429,3 \mathrm{~km}^{2}$ de área total. Dentre as principais atividades econômicas no município tem-se a pesca (principalmente de lagosta), extrativismo do coco, coleta de algas e mariscos, agricultura de subsistência, artesanato, beneficiamento da castanha de caju, beneficiamento de pescado e carcinicultura (Nascimento, 2006).

A praia de Picos $\left(04^{\circ} 39^{\prime} 46^{\prime \prime} \mathrm{S} / 37^{\circ} 26^{\prime} 25^{\prime \prime} \mathrm{O}\right)$ é uma enseada que apresenta extensão média de $2,5 \mathrm{~km}$. Possui planície litorânea com declive suave para o mar, um largo estirâncio, presença de arenitos de praia (beachrocks) e é limitada, no supra litoral, por falésias do grupo Barreiras (Alves, 2007).

A regiáo de Icapuí possui atributos de biodiversidade e serviços ambientais que levaram o Ministério do Meio Ambiente a considerá-la como de importância biológica Extremamente Alta, com prioridade de ação também Extremamente Alta (Ministério do Meio Ambiente, 2007), onde se recomenda a criação de uma AMP.

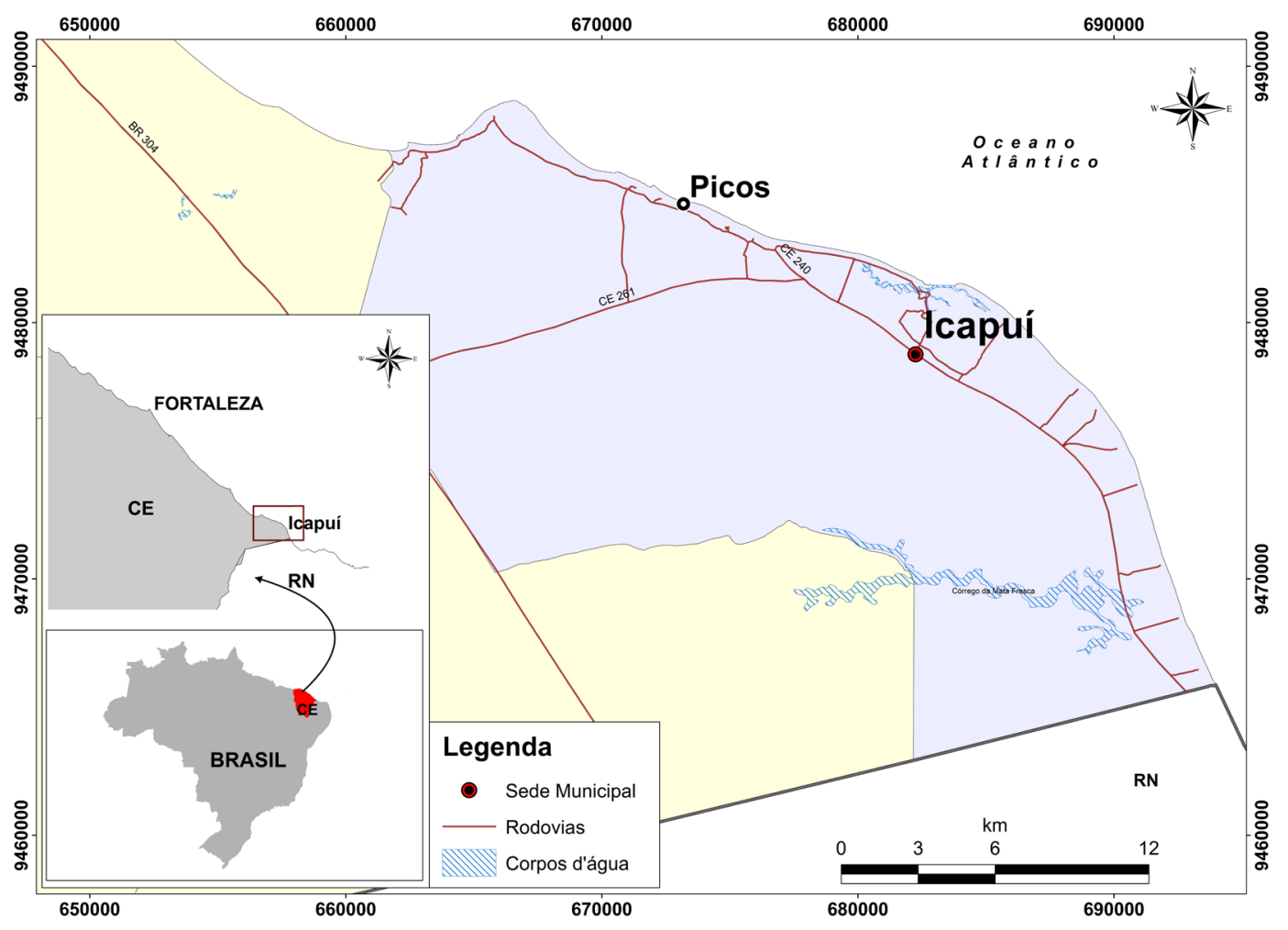

Figura 1. Localização da área de estudo (Praia de Picos, Estado do Ceará, Nordeste do Brasil). Figure 1. Location of the study site (Picos Beach, Ceará State, Northeast of Brazil). 


\subsection{Metodologia de amostragem}

Para este estudo, questionários semi-estruturados foram elaborados (Anexo 1) para realização de entrevistas com moradores na comunidade de Picos, Icapuí-CE, no período de maio e junho de 2012. A comunidade de Picos é relativamente pequena, possui 36 casas, e dessas, 10 são casas de veraneio ou sem habitação. As 26 casas de moradores totalizam aproximadamente 100 pessoas, sendo 50 adultos. A contagem foi realizada pelos pesquisadores no período das entrevistas. Foram visitadas 20 casas $(80 \%$ do total de residentes) e entrevistados 30 moradores $(60 \%$ do total da comunidade), sendo 15 homens e 15 mulheres. O questionário foi realizado oralmente e as respostas foram registradas de forma escrita. Antes das entrevistas explicou-se o motivo da pesquisa e todos os moradores concordaram em participar e permitiram a divulgação das respostas. Buscouse caracterizar sócio-economicamente a comunidade, listar os componentes da fauna local existentes e descrever os recursos ambientais utilizados pelos moradores. Ainda, foi investigado o interesse da população em criar uma AMP na regiâo.

A identificação das espécies de peixe e a relação com os nomes populares utilizados em Icapuí foi registrada por Salles (2011), baseada em literatura especializada (Menezes; Figueiredo, 1985; Cervigón, 1996; Gadig et al., 2000).

Foi realizada uma oficina com quatro pescadores nativos para elaboração de um mapa ambiental da área de estudo, identificando características sedimentológicas da praia, locais de ocorrência de algas e fanerógamas marinhas, recifes areníticos e fontes de água doce. Essa oficina foi desenvolvida na residência de um dos pescadores, no topo da falésia, onde havia vista para toda a área de estudo. Os pescadores foram selecionados pelo tempo de pesca (acima de 50 anos) e apurado conhecimento da área segundo indicaçóes de moradores e de membros da Associação de Pesquisa e Preservação de Ecossistemas Aquáticos - Aquasis, que realiza pesquisas na região há cerca de 15 anos com o apoio de colaboradores locais. Os pescadores fizeram o desenho da zona costeira com uma corda e foram inserindo no esquema rochas, plantas e outros utensílios do local para delimitar proporcionalmente as feições e características ambientais da região. A partir da maquete, elaborou-se um mapa desenhado à máo com o auxílio dos pescadores e, posteriormente, foi elaborado um mapa digital baseado na percepção ambiental destes. Realizaram-se também mergulhos livres na área de estudo para observar a biodiversidade local e averiguar a compatibilidade das informaçóes passadas pela comunidade.

\section{RESULTADOS E DISCUSSÃO}

\subsection{Pescadores artesanais: caracterizaçáo sócio- econômica e principais recursos biológicos e pesqueiros}

A idade média entre os entrevistados foi de 32 anos $( \pm$ 17 anos), sendo a mínima 20 e a máxima 84. Dentre os entrevistados, vinte eram moradores nativos $(66,6 \%)$ e dez não nativos. O tempo de moradia médio na região foi de 26 anos ( \pm 20 anos), sendo o mínimo 5 meses e o máximo 66 anos.
Com relação ao perfil socioeconômico, a profissão mais citada foi pescador $(43,3 \%)$, seguida de dona de casa e agricultor. Dentre os homens, $87 \%$ são pescadores e dentre as mulheres, $74 \%$ são donas de casa. Constatou-se, portanto, que a comunidade é essencialmente pesqueira; e a agricultura, principalmente de subsistência, é uma atividade paralela importante na comunidade. Outras profissóes citadas em minoria foram: artesão, pedreiro, vigilante e estudante.

O principal recurso pesqueiro procurado pelos pescadores é a lagosta (Panulirus argus e Panulirus laevicauda). Dentre os crustáceos, eles também pescam camaróes dos gêneros Xiphopenaeus, Farfantepenaeus e Litopenaeus e coletam diversos tipos de siris e caranguejos (Infraordem Brachyura). Na regiāo, são comumente capturadas ao longo do ano diversas espécies de peixes (Figuras 2 e 3), como: agulha-preta (Hemiramphus brasiliensis), bagre (Cathorops spixii), boca-mole (Larimus breviceps), biquara (Haemulonplumieri), canguito (Orthopristis ruber), carapeba (Diapterus sp.), camurim (Centropomus sp.), camurupim (Megalops atlanticus), coró amarelo (Conodon nobilis), cururuca (Micropogonias furnieri), dentão (Lutjanus jocu), galo (Selene sp.), galo do alto (Alectis ciliaris), garajuba (Caranx sp.), judeu (Menticirrhus americanus), mariquita (Holocentrus adscensionis), pescada (Cynoscion sp.), pirambu (Anisotremus surinamensis), serra (Scomberomorus brasiliensis) e tibiro (Oligoplistes saliens), dentre outros. Estas espécies também foram comentadas por Almeida (2010), Marinho (2010) e Salles (2011).

Os dados obtidos por este estudo estão de acordo com a literatura, a qual observa que a pesca artesanal na região contempla tanto as capturas com fins de subsistência, associado à obtenção de recursos alimentares para o sustento das próprias famílias, como as capturas com o objetivo essencialmente comercial. As unidades familiares ou grupo de vizinhança, sem a prática do assalariamento, são maioria como módulo de pesca. As embarcaçóes são geralmente de pequeno porte, de propulsão natural (vela, remo ou vara) ou motorizadas, sendo utilizados métodos diversos de captura, de natureza artesanal e comumente confeccionados pelos próprios pescadores, que resultam em capturas multiespecíficas (Almeida, 2010; Marinho, 2010; Salles, 2011; Barroso, 2012).

Segundo as respostas obtidas nas entrevistas, observouse que alguns dos peixes são capturados ainda em estado juvenil na região dos recifes areníticos (Figura 4). Crustáceos e moluscos também são encontrados em fase inicial de vida nas capturas para consumo, ressaltando a importância da região como berçário para diversas espécies (Almeida, 2010; Marinho, 2010; Barroso, 2012).

Ainda segundo os moradores, a praia é local de ocorrência de peixe-boi marinho (Trichechus manatus), tartaruga verde (Chelonia mydas), tartaruga de pente (Eretmochelys imbricata) e o peixe mero (Epinephelus itajara), espécies classificadas em alguma categoria de ameaça de extinção na Lista Vermelha da IUCN - International Union for Conservation of Nature, e incluídas na Lista Nacional das Espécies da Fauna Brasileira Ameaçadas de Extinção, do Ministério do Meio Ambiente.

De acordo com a IUCN, o peixe boi é uma espécie ameaçada de extinção, e segundo Alvez (2007), utiliza a praia de picos pra se alimentar e reproduzir. 

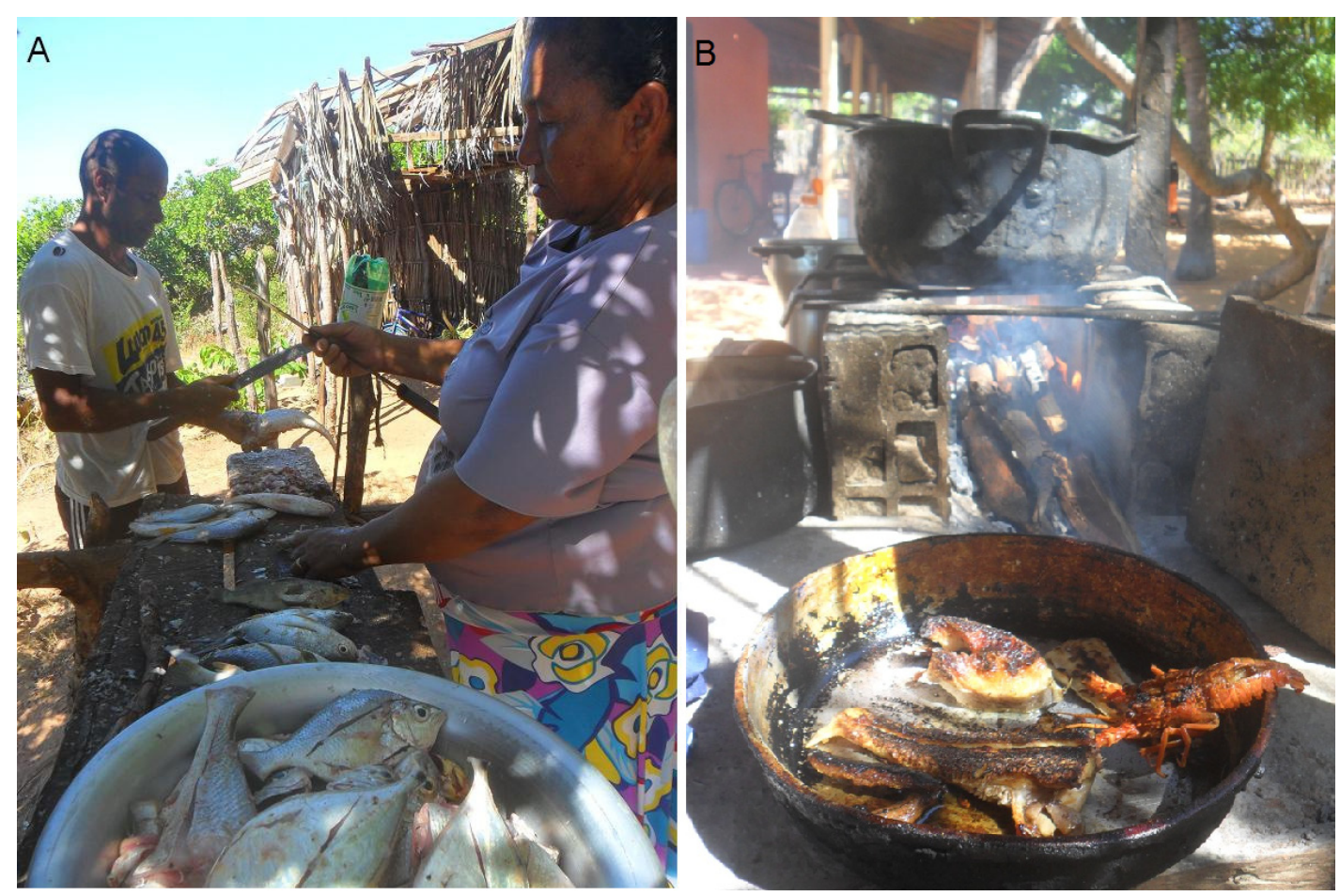

Figura 2. a) Moradores locais beneficiando o pescado para consumo da família; b) Formas de preparo e consumo dos pescados.

Figure 2. a) Local residents benefiting fish for family consumption; b) Methods of preparation and consumption of fishing resources; foods are cooked in wood-fired oven and are quite consumed roasted, besides cooked.
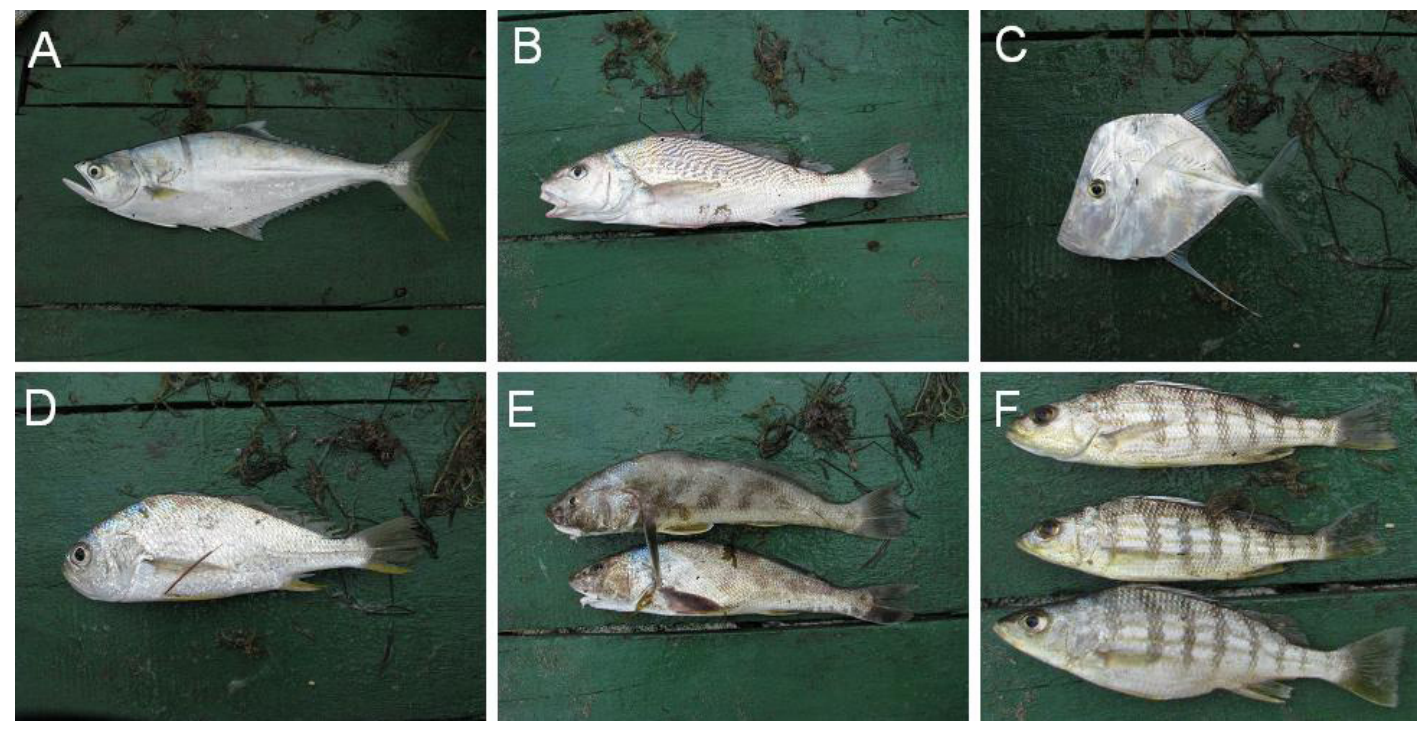

Figura 3. Peixes capturados por rede de rengalho na Praia de Picos: a) Tibiro: Oligoplistes saliens; b) Cururuca: Micropogonias furnieri; c) Galo: Selene sp.; d) Boca mole: Gênero breviceps; e) Judeu: Menticirrhus americanus; f) Coró amarelo: Conodon nobilis.

Figure 3. Fish caught by rengalho net in Picos Beach. a) Tibiro: Oligoplistes saliens b) Cururuca: Micropogonias furnieri c) Galo: Selene sp. d) Boca mole: breviceps e) Judeu: Menticirrhus americanusf) Coró amarelo: Conodon nobilis. 

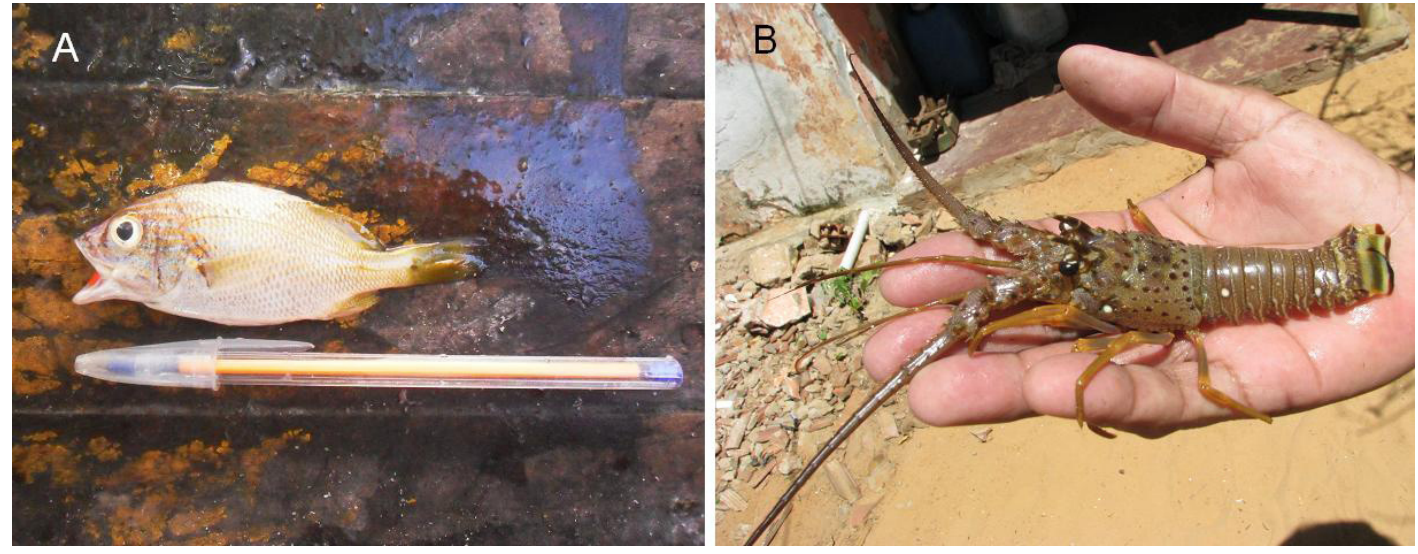

Figura 4. a) Biquara (Haemulon plumieri) juvenil capturada com linha e anzol nos recifes areníticos da área de estudo; b) Lagosta (Panulirus spp.) juvenil capturada em marambaia.

Figure 4. a) Juvenile Biquara (Haemulon plumieri) caught by hook and line in the sandstone reefs in the study area; b) Lobster (Panulirus spp.) juvenile captured in Marambaia.

\subsection{Percepçáo ambiental e recursos}

Observa-se que os pescadores possuem conhecimento apurado sobre as características ambientais da regiáo, tanto em relação à localização, associação entre recursos e substratos, quanto à disponibilidade e sazonalidade dos recursos (Figura 5). Os dados da percepçáo ambiental dos pescadores quanto a aspectos da ecologia de peixes e quanto a processos morfodinâmicos foram condizentes com a literatura científica disponível para a área (Fonteles-Filho et al., 1988; Ivo \& Pereira, 1996; Alves, 2007; Aquasis, 2011; Salles, 2011). Este fato também foi notado por diversos autores (Berkes, 1993; Agardy, 2000; Gerhardinger et al., 2009; Glaser et al., 2010; Areizaga et al. 2012), que reforçam a efetividade das açôes de manejo quando a população local é envolvida nos processos de gestão.

Ao longo da plataforma continental da regiáo existem variados substratos e ecossistemas submersos que desempenham papéis fundamentais na manutenção da biodiversidade e da produtividade das águas costeiras, como bancos submersos de algas e fanerógamas, substratos de algas calcáreas, fundos de lama biodetrítica e afloramentos rochosos que emergem na maré baixa, desde a plataforma interna, passando pela zona de estirâncio ao sopé das falésias (Alves, 2007). A vegetação de tabuleiro localizada no topo e encostas das falésias é responsável pela diminuição dos efeitos da erosão. No caso da área de estudo, predominam as fácies carbonáticas e de areias quartzosas. A geologia, clima e drenagem dos rios propiciam um substrato composto por algas calcárias e demais sedimentos biodetríticos (Morais \& Freire, 2003), conforme também apontado pelos resultados da percepção ambiental. Os bancos de algas apresentam associaçôes entre diversas espécies de algas, principalmente algas vermelhas (Rhodophyta). Em relação às fanerógamas marinhas, são encontradas na região espécies do gênero Halodule, conhecida como capim-agulha. Estes ambientes também foram demonstrados no mapa dos ambientes costeiros delineado pelos pescadores artesanais, e do gênero Halophila, as quais foram visualizadas em mergulhos na área.
Os dados geológicos de Alves (2007) e Almeida (2010) para a região corroboram o mapa (referente aos tipos de fácies sedimentares), elaborado pelos pescadores.

Os bancos de algas e os recifes são intensamente explorados em todo o mundo devido à riqueza biológica e a proximidade da costa. Contudo, a preservação desses ecossistemas é fundamental para a reposição do estoque de juvenis de lagostas. Nas áreas de berçário, os puerulus de lagosta $P$. argus fixam-se, preferencialmente, em habitat de estrutura complexa, tal como a alga vermelha (Eggleston et al., 1992; Cruz et al., 2011; Barroso, 2012).

A formação geológica Barreiras se apresenta exposta em falésias na zona costeira da praia da área de estudo sendo responsável pelo acúmulo de água, que verte em afloramentos submersos. Segundo os pescadores, esses afloramentos, chamados "olheiros" ou "olhos d'água", mudam de posição ao longo dos anos devido a dinâmica sedimentar, mas geralmente estão próximos aos arenitos de praia, feição formada na face de praia por cimentação por carbonato de cálcio (Guerra \& Cunha, 1998). A disponibilidade de água doce próximo à costa e a abundância de algas marinhas e capim agulha favorecem ao ciclo de vida de muitas espécies de importância econômica e ecológica, como camarôes dos gêneros Xiphopenaeus, Farfantepenaeus e Litopenaeus; lagostas do gênero Panulirus e o peixe-boi marinho, Trichechus manatus. Este tópico foi abordado com ênfase pelos pescadores no mapa supracitado, observando a importância da conservação deste ambiente.

Muitos países carecem de informação científica sobre ecologia de peixes e o conhecimento dos pescadores vem a ser uma fonte importante para melhorar o conhecimento sobre ecologia, migração, reprodução, hábitos alimentares e mudanças na abundância de um conjunto diversificado de recursos pesqueiros (Silvano \& Begossi, 2012). Estas abordagens junto ao conhecimento popular são importantes para obter um manejo das espécies e da pesca como um todo, considerando a abordagem ecossistêmica (Batista et al., 2011). De fato, as experiências destes pescadores artesanais que habitam a localidade de Picos ou proximidades há 


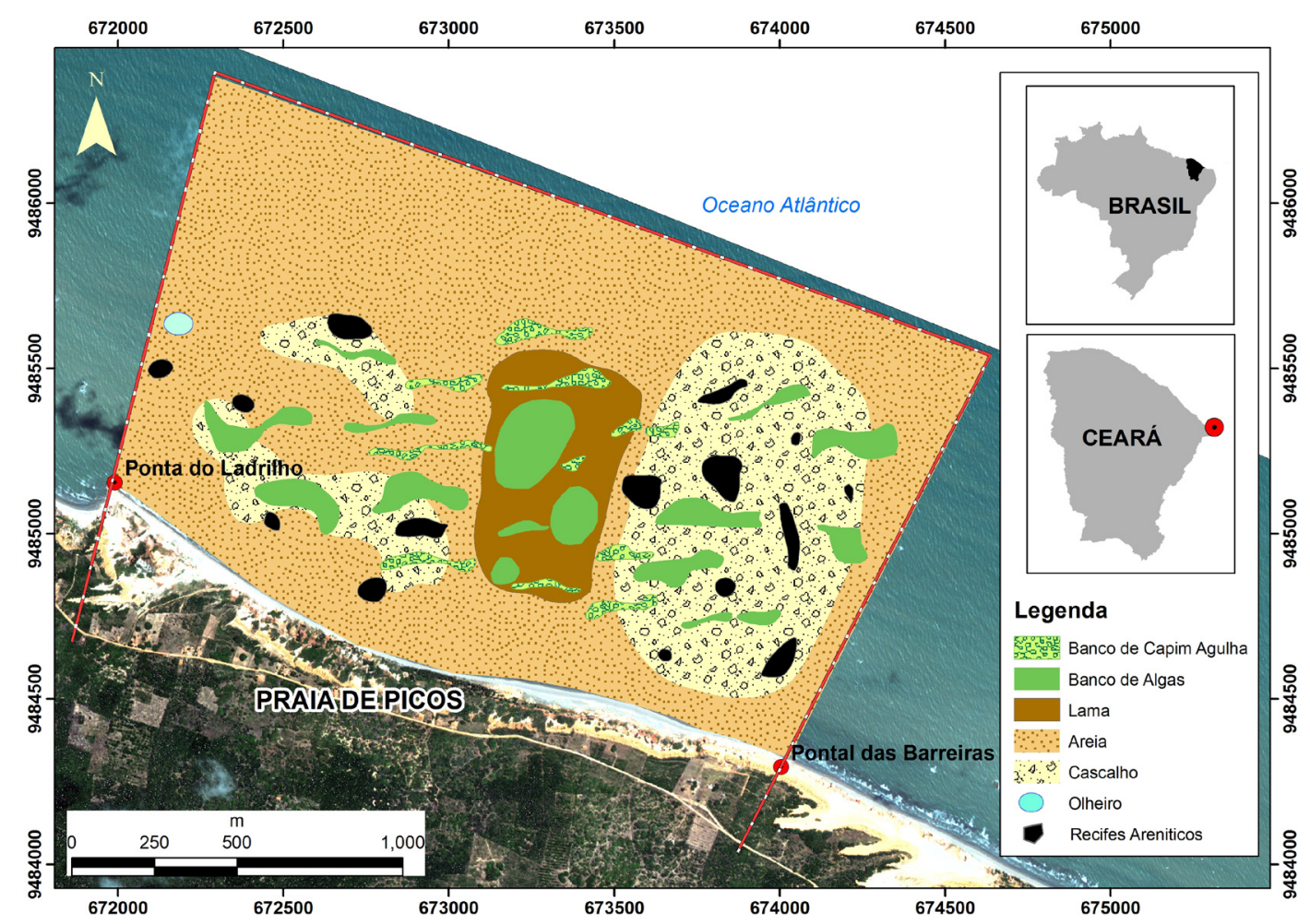

Figura 5. Mapa da praia de Picos, desenvolvido em oficina com pescadores nativos, indicando os recifes areníticos, olheiro, bancos lamosos e locais de bancos de capim agulha e algas.

Figure 5. Map of Picos beach indicating reefs, scout, muddy banks and local banks needle grass and algae. The map was developed from a workshop with native fishermen.

pelo menos cinco geraçôes, devem ser consideradas para medidas de gestão pesqueira e/ou ecossistêmica na regiáo, principalmente por ser a criação de uma AMP uma demanda local.

Além da participação na gestáo, é importante que a comunidade seja envolvida nas etapas de zoneamento e categorizaçáo daárea, e isso deveestar atrelado ao levantamento das características socioambientais através do conhecimento dos usuários dos recursos, evitando mecanismos de gestáo top-down (Glaser et al., 2010).

Nos últimos anos, estudos científicos sobre a pesca artesanal têm utilizado como fonte de informação os próprios pescadores locais, devido às suas ricas experiências acumuladas, como exemplificado pelo estudo de Batista et al. (2011) para uma área marinha protegida em Portugal. Esses indivíduos possuem uma amplitude de saberes e crenças transmitidas culturalmente através de geraçôes sobre a relaçáo da biosfera (incluindo os humanos) com as variáveis ambientais e oceanográficas (Berkes, 1993; Suuronen et al., 2010).

Os saberes dos entrevistados na área de estudo englobam diversos campos como a sistemática das espécies aquáticas, o comportamento da ictiofauna, taxonomia, padrōes reprodutivos, processos de migração das espécies e as teias tróficas. Observa-se assim, como os dados inventariados nesta pesquisa, que são comuns na literatura os relatos de compreensão profunda sobre as características físicas e geológico-atmosféricas (nuvens, ventos, mudança de tempo), as artes de navegaçáo e, sobretudo, da dinâmica dos recursos pesqueiros. Batista et al. (2011) observaram a importância do conhecimento local para entendimento do funcionamento do ambiente, redução de conflitos e tomada de decisão mais participativa. Rios et al. (2007) abordaram que conjugar o conhecimento científico ao conhecimento tradicional dos pescadores artesanais pode ser uma valiosa ferramenta no manejo da atividade pesqueira de pequena escala, uma vez que a realidade pesqueira pode ser melhor retratada. Scholz et al. (2004) demonstraram que o conhecimento local dos pescadores, dentro de uma abordagem geoespacial, é imprescíndivel para o planejamento das áreas marinhas protegidas, que visam desenvolver sustentavelmente a pesca e adotar mecanismos participativos para a tomada de decisão.

\subsection{Desenvolvimento sustentável da regiáo e proposiçáo de áreas marinhas protegidas}

Em relação à percepçáo das mudanças socioeconômicas ocorridas na comunidade, cerca de $45 \%$ dos entrevistados considera que a qualidade de vida das famílias melhorou ao longo dos anos, e cerca de $45 \%$ considera que piorou. Os 10\% restantes consideram que não houve mudanças significativas. Contudo, os valores se alteram se considerarmos em separado a opinião de nativos e não nativos. A maioria dos nativos acha que a qualidade de vida melhorou, atribuindo esta melhora à disponibilidade de água canalizada e energia, transporte e 
auxílios do governo. Já a piora é atribuída, principalmente, aos problemas associados à pesca. Essa diferença entre as percepçóes pode estar relacionada ao fato de que os nativos possuem uma perspectiva mais antiga da vida da comunidade, analisando as mudanças desde o tempo de seus pais e avós.

Quase todos os entrevistados (97\%) afirmaram gostar de morar em Picos devido à tranquilidade, disponibilidade de alimento, beleza cênica, natureza, convivência com os outros moradores e reuniōes religiosas. Porém, também foram citados diversos pontos negativos: problemas associados à pesca, como escassez da lagosta, prática da pesca de compressor, falta de fiscalização, utilização de pesca com estruturas inadequadas fundeadas como atratores (marambaias), que diminuem a qualidade do pescado e são motivo de conflitos com pescadores de outras comunidades; falta de emprego, descaso da prefeitura em relação à saúde; falta de oferta de cursos para os jovens; poucos meios de transporte; difícil acesso à praia; pouco turismo; e falta de algumas áreas preservadas, como as falésias da regiáo (Tabela 1).

As marambaias são recifes artificiais, atualmente feitos com tambores cilíndricos de ferro, pneus, eletrodomésticos e outros objectos que servem para agregaçáo de lagostas, facilitando a captura através do mergulho com compressor. $\mathrm{O}$ excessivo número de marambaias particulares está limitando o acesso às áreas de pesca e, de certa forma, "loteando" o espaço marítimo da plataforma continental, pois o pescador que investiu para construí-las considera que tem direitos exclusivos sobre os recursos pesqueiros nela existentes (Salles, 2011). Além de ilegal pela legislação brasileira, a pesca com compressor é feita de maneira perigosa, onde os mergulhadores respiram através uma mangueira conectada a um bujão de gás de cozinha munido de oxigênio e acoplado ao motor da embarcação. Os pescadores muitas vezes não possuem conhecimento das técnicas e medidas de segurança no mergulho, muitos sofrem doenças descompressivas e vários já morreram, segundo relatos locais. Os pescadores artesanais alegam que a pesca com compressor é o motivo da diminuição da produção pesqueira artesanal e devido a conflitos entre os mergulhadores e os pescadores artesanais, a comunidade de Icapuí encontra-se na chamada guerra da lagosta desde a década de 80 , a qual já gerou inúmeras mortes (Marinho, 2010).

Nos últimos anos, a pesca irregular e indiscriminada de várias espécies de importância econômica, incluindo a lagosta, vem proporcionando a diminuição dos estoques e da efetividade das capturas pela pesca artesanal. Como conseqüência, temos a diminuição da renda de comunidades pesqueiras que, por sua vez, estimula a sobrepesca e a captura de indivíduos imaturos (Nascimento, 2006).

Pesquisas revelam que o esforço de pesca empregado na captura da lagosta no Brasil se encontra, desde 1972, em níveis superiores ao considerado ótimo para a captura máxima sustentável (Fonteles-Filho et al., 1988; Silva \& Rocha, 1999; Barroso, 2012). A produção lagosteira na região analisada (Icapuí, NE Brasil) acompanha a tendência de produção da regiáo costeira do estado do Ceará, caracterizada por um período de oscilação (2001-2006), com uma queda acentuada na produção em 2006. Neste período, a região de estudo (município) produziu em média, 284 toneladas de lagosta por ano. $\mathrm{O}$ declínio dos estoques pesqueiros de lagosta está intimamente correlacionado com o excesso de esforço de pesca, tanto de natureza legal quanto ilegal, e com as atividades de pesca ilegal, onde são comercializados indivíduos imaturos (Ivo \& Pereira, 1996; Marinho 2010; Salles, 2011; Barroso, 2012). A comunidade analisada faz parte do grupo de comunidades que ainda sustentam a pesca artesanal (feita através de embarcaçóes a velas, redes e armadilhas - manzuás) no litoral brasileiro e a cada ano apresenta menor produção pesqueira (Salles, 2011). A região encontra-se em situação emergencial para a gestão pesqueira. Como medidas de manejo, outras atividades econômicas podem ser incentivadas, de acordo ao interesse dos envolvidos, visando a geração de renda complementar e a diminuição dos conflitos neste setor, como o cultivo de algas e peixes, extração de outros recursos e a agricultura. Cerca de $83 \%$ dos moradores de Picos utilizam a agricultura como fonte paralela de renda e subsistência, plantando espécies como feijão, milho e frutas tropicais como melancia, caju e coco. Praticam principalmente agricultura de subsistência e dependem de águas pluviais para manutenção das culturas, pois nos roçados não possuem poço, cisterna ou água encanada. Os pescadores artesanais náo raro pertencem a comunidades que possuem histórico de auto-suficiência para muitas de suas necessidades, tais como alimentos e remédios, assim como observado em outras regiôes do Brasil (Hanazaki et al., 2000).

De acordo com a pesquisa realizada, observa-se o aceite de implantação de AMP e gestão dos recursos visando ao manejo sustentável da pesca, objetivando-se recuperar os estoques pesqueiros e assegurar a sustentabilidade das capturas no futuro. Além disso, todos os entrevistados afirmaram ter interesse na implantação de uma área marinha protegida na região (como uma reserva extrativista marinha) e que estariam dispostos a colaborar com a gestão da mesma (auxiliando em mais estudos de caracterização, etapas de zoneamento, a elaboração do plano gestor e comprometendo-se em cumprir

Tabela 1. Quadro geral sobre os pontos positivos e negativos, mais citados nas entrevistas, da comunidade pesqueira. Table 1. Positive and negative aspects most cited in interviews with the fishing community.

\begin{tabular}{|c|c|}
\hline Pontos positivos & Pontos negativos \\
\hline $\begin{array}{l}\text { - Tranquilidade } \\
\text { - Biodiversidade } \\
\text { - } \text { Beleza cênica } \\
\text { - } \text { Disponibilidade de recursos }\end{array}$ & $\begin{array}{l}\text { - Problemas associados à pesca } \\
\text { - Falta de emprego } \\
\text { - Má infraestrutura } \\
\text { - Descaso da prefeitura }\end{array}$ \\
\hline
\end{tabular}


as normas definidas para conservação da área), corroborando o modelo de área marinha protegida baseada em uma gestáo comunitária (bottom-up).

Voyer et al. (2012), ao descreverem a participação pública em três casos de áreas marinhas protegidas na Austrália, mostraram que nos casos de sucesso na implementaçáo e gestão de AMPs houve alto nível de engajamento e compromisso da comunidade para com o poder público. Trenouth et al. (2012), em estudo sobre áreas marinhas protegidas também realizado naquele país, abordaram que as comunidades e sua percepção podem ser extremamente úteis para avaliar os riscos ambientais e a efetividade do manejo.

Gerhardinger et al. (2009), em estudo com nove áreas marinhas protegidas no Brasil demonstraram que apesar da importância do conhecimento ecológico tradicional, a abordagem utilizada é essencialmente científica e não dialógica com os saberes populares dos pescadores artesanais. Bogaert et al. (2009) ao relatarem o histórico de criação das áreas marinhas protegidas na Bélgica encontrou abordagem semelhante no início do século XX, com pouca participação pública.

A percepçáo dos pescadores pode ser usada para o planejamento de AMPs, inclusive devido informaçôes sobre a migração dos recursos pesqueiros (Suuronen et al., 2010). Uma abordagem semelhante à usada no presente estudo foi citada por Read et al. (2011), acerca da importância do conhecimento local na construçáo de zoneamentos ambientais para AMPs. Areizaga et al. (2012) comentando sobre a eficiência da gestão costeira integrada, utilizou de abordagem sistêmica baseada no conhecimento popular, para a classificação de sistemas ambientais no litoral da Espanha.

Propóe-se, na região analisada, incluindo a região dos ambientes recifais, as falésias e a vila de moradores, a criação de uma AMP de Uso Sustentável que tem como objetivo manter os ecossistemas naturais e regular o uso admissível da área, de modo a compatibilizá-lo com os objetivos de conservação, como uma reserva extrativista ou uma Área de Relevante Interesse Ecológico. A área em questão faz parte de uma regiáo mais abrangente na qual já foram propostas anteriormente criaçóes de AMPs.

Marinho (2010) em pesquisa na regiâo do litoral leste do Ceará observou que uma soluçáo da crise pesqueira seria um modelo de co-gestáo do ordenamento pesqueiro, por meio da criação de uma AMP com $2.200 \mathrm{~km}^{2}$, a qual beneficiaria 13 comunidades pesqueiras pertencentes aos municípios de Aracati e Icapuí, num total aproximado de 1930 pescadores, incluindo os da área analisada neste estudo. Entretanto, naquela abordagem os pescadores ainda náo haviam sido consultados em relação à criação de uma AMP e nem foram envolvidos em etapas de implementação. Voyer et al. (2012) argumentaram que a consideraçáo dos diversos pontos de vista, incluindo os grupos minoritários como os pescadores artesanais, é essencial para garantir uma abordagem equitativa e socialmente justa para a criação e gerenciamento de áreas marinhas protegidas.

Ângulo-Valdes \& Hatcher (2010) analisaram uma nova classificação das AMPs, onde se observa que a abordagem proposta para esta área no Nordeste Brasileiro traz benefícios para a comunidade (diretos e indiretos) e para os ecossistemas, dentro do conceito holístico das AMPs. Ban et al. (2011) em estudo sobre as AMPs com ambientes recifais, afirmam que o primeiro paradigma a ser alcançado para a efetividade destas áreas é o envolvimento e conhecimento da comunidade. Areizaga et al. (2012) abordaram que o envolvimento das comunidades, através de uma participação efetiva, é um dos principais objetivos das agendas governamentais com transparência, honestidade e com altos níveis de desempenho em termos de gestâo costeira integrada.

Suuronen et al. (2010) demonstraram que, na ausência de dados consistentes sobre os recursos e o tipo adequado de AMP segundo o planejamento participativo, há grande probabilidade de ineficiência no estabelecimentos destas áreas marinhas na conservação de estoques pesqueiros. Scholz et al. (2004) abordaram que normalmente os pescadores são contrários a AMPs, nas quais se restrinja o acesso total aos recursos pesqueiros (em prol do turismo ou da preservaçáa dos recursos). Entretanto, no caso de AMPs que visem desenvolver de modo sustentável práticas de pesca tradicional, muitas vezes, de grande importância sócioeconômica e na manutenção da cultura, ocorre aceitação por parte da comunidade pesqueira.

Recomenda-se que a unidade de conservação a ser criada, baseie-se num modelo participativo e que seja incluído no Plano de Manejo, além de ações conservacionistas, açôes comunitárias de ecoturismo e de agricultura familiar planejada, visando a valorização ambiental, diversificação da atividade econômica e geração de renda local, sempre em parceria com as comunidades e de acordo aos interesses comuns da maioria dos agentes locais.

\section{CONSIDERAÇÓES FINAIS}

A gestão eficiente das áreas marinhas protegidas, em geral, necessita de uma gestáo participativa desde a sua criação. No contexto das áreas costeiras, os pescadores artesanais podem dar uma grande contribuição para o gerenciamento destes ambientes, incluindo a implementação de unidades de conservação, através do compartilhamento das suas experiências sobre a estrutura e o funcionamento dos ambientes marinhos; além disto, o modelo de gestão comunitária deve incluir universidades, organizaçôes nãogovernamentais, poder público e movimentos sociais. A percepção ambiental dos pescadores aplicados ao zoneamento ambiental $\mathrm{e}$ ao entendimento do funcionamento dos serviços ambientais em áreas protegidas pode ser uma grande contribuição na busca da gestão integrada da zona costeira.

\section{AGRADECIMENTOS}

Aos moradores da praia de Picos (Icapuí-Ceará) pela colaboração com a pesquisa, à Universidade Federal do Ceará pelo incentivo e à Organizaçáo Náo Governamental (ONG) Associação de Pesquisa e Preservação de Ecossistemas Aquáticos (Aquasis) pela disponibilização de bibliografia e dados sobre a área em questão. 


\section{REFERÊNCIAS BIBLIOGRÁFICAS}

Agardy, M.T. (1994) - Advances in Marine Conservation: The Role of Marine Protected Areas. Trends in Ecology \& Evolution, 9(7):267-270. DOI:10.1016/01695347(94)90297-6

Agardy, M.T. (2000) - Information Needs For Marine Protected Areas: Scientific and Societal. Bulletin OfMarine Science (ISSN: 0007-4977), 66(3):875-888. Disponível em http://coral.geog.uvic.ca/cisdemo/sites/default/files/ Agardy_information\%20needs\%20MPAs.pdf

Almeida, L.G. (2010) - Caracterização das áreas de pesca de lagosta na praia da Redonda, Icapui-CE. 93p., Dissertaçáo de Mestrado, Universidade Federal do Ceará, Fortaleza, CE, Brasil. Disponível em http://www.repositorio.ufc. br:8080/ri/bitstream/123456789/1925/1/2010_dis_ lgdealmeida.pdf 10.1016/0169-5347(94)90297-6

Alves, M.D.O. (2007) - Peixe-Boi Marinho, Trichechus manatus manatus: Ecologia e Conhecimento Tradicional no Ceará e Rio Grande do Norte, Brasil. 118 p., Dissertação de Mestrado, Universidade Federal de Pernambuco, Recife, PB, Brasil. Não publicado.

Areizaga, J.; Sanò, M.; Medina, R.; Juanes, J. (2012) - Improving public engagement in ICZM: A practical approach. Journal of Environmental Management, 109:123-135. DOI: 10.1016/j.jenvman.2012.05.006

Ban, N.C.; Adams, V.M.; Almany, G.R.; Ban, S.; Cinner, J.E.; McCook, L.J.; Mills, M.; Pressey, R.L.; White, A. (2011) - Designing, implementing and managing marine protected areas: Emerging trends and opportunities for coral reef nations. Journal of Experimental Marine Biology and Ecology, 408(1-2):21-31. DOI: 10.1016/j. jembe.2011.07.023

Barroso, J.C. (2012) - Avaliação da pesca da lagosta vermelha (Panulirus argus) e da lagosta verde (Panulirus laevicauda) na plataforma continental do Brasil. 109p., Dissertação de Mestrado, Universidade Federal do Ceará, Fortaleza, CE, Brasil. Não publicado.

Batista, M.I.; Baeta, F.; Costa, M.J.; Cabral, H.N. (2011) - MPA as management tools for small-scale fisheries: The case study of Arrábida Marine Protected Area (Portugal). Ocean \& Coastal Management, 54(2):137147. DOI:10.1016/j.ocecoaman.2010.10.032

Begossi, A. (2004) - Ecologia de pescadores da Mata Atlântica e Amazônica. 332p., Hucitec Editora, São Paulo, SP, Brasil. ISBN:8527106248.

Berkes, F. (1993) - Traditional knowledge in perspective. In: Julian T. Inglis (org.), Traditional knowledge: concepts and cases,pp.1-10, InternationalDevelopmentResearch Centre (IDRC), Ottawa, Ontario, Canada. ISBN: 0-88936683-7. Disponível em http://books.google.com.br/books ?id=J2CNS64AFvsC\&lpg=PA1 \&ots=KzilFkqkMr\&dq= Berkes\%2C\%20F.\%20(1993)\%20-\%20Traditional\%20 knowledge $\% 20$ in $\% 20$ perspective $\& l r=l a n g \_e n \& h l=p t-$ $\mathrm{BR} \& \mathrm{pg}=\mathrm{PP} 1 \# \mathrm{v}=$ onepage \&q\&f$=$ false

Bogaert, D.; Cliquet, A.; Maes, F. (2009) - Designation of marine protected areas in Belgium: A legal and ecological success?. Marine Policy, 33(6):878-886. DOI: 10.1016/j. marpol.2009.04.020
Cervigón, F.M. (1996) - Los peces marinos de Venezuela. 254p., Fundación Científica Los Roques, Caracas, Venezuela. ISBN: 980-6028-17-1

Cinner, J.E.D. (2007) - Designing marine reserves to reflect local socioeconomic conditions: lessons from longenduring customary management systems. Coral Reefs, 26(4):1035-1045. DOI: 10.1007/s00338-007-0213-2

Cruz, R.; Conceição, R.N. de L.; Marinho, R.; Barroso, J.C.; Holanda, J.S.; Félix, C.S.; Martins, M.E.O.; Santos, F.S.; Araújo Silva, K.C.; Furtado-Neto, M.A.A.; Pereira da Costa, F.A.; Garcez, D.S.; Torres, M.T. (2011) Metodologias de amostragem para avaliação das populaçóes de lagosta: plataforma continental do Brasil. 142p., UFC / LABOMAR / NAVE, UH, CIM, Fortaleza, CE, Brasil. ISBN: 978857563872

Eggleston, D.B.; Lupclus, R.N.; Miller, D.L. (1992) Artificial shelters and survival of juvenile Caribbean spiny lobster Panulirus argus: Spatial, habitat, and lobster size effects. Fishery Bulletin (ISSN: 0090-0656), 90(4):691702. Disponível em http://fishbull.noaa.gov/904/ eggleston.pdf

Fonteles-Filho, A.A; Ximenes, M.O.C.; Monteiro, P.H.M. (1988) - Sinopse de informaçóes sobre as lagostas Panulirus argus (Latreille) e P. laevicauda (Latreille) (Crustacea: Palinuridae), no Nordeste do Brasil. Arquivo Ciências do Mar (ISSN 0374-5686), 27:1-19, Fortaleza, CE, Brasil. Disponível em http://www.labomar.ufc. br/images/stories/arquivos/ArqCienMar/V27_1988/ acm_1988_27_1_01.pdf

Gadig, O.B.F.; Bezerra, M.A. Feitosa, R.D.; Furtado-Neto, M.A.A. (2000) - Ictiofauna marinha do Ceará, Brasil: I. Elasmobranchii. Arquivos de Ciências do Mar (ISSN 03745686), 33:127-132, Fortaleza, CE, Brasil. Disponível em http://www.labomar.ufc.br/images/stories/arquivos/ ArqCienMar/V33_2000/acm_2000_33_17.pdf

Game, E.T.; Bode, M.; McDonald-Madden, E.; Grantham, H.S.; Possingham, H.P. (2009) - Dynamic marine protected areas can improve the resilience of coral reef systems. Ecology Letters, 12(12):1336-1346. DOI: 10.1111/j.1461-0248.2009.01384.x

Gerhardinger, L.C.; Godoy, E.A.S.; Jones, P.J.S. (2009) - Local ecological knowledge and the management of marine protected areas in Brazil. Ocean \& Coastal Management, 52(34):154-165. DOI :10.1016/j.ocecoaman.2008.12.007

Glaser, M.; Baitoningsih, W.; C.A. Ferse, S. C. A.; Neil, M.; Deswandi, R. (2010) - Whose sustainability? Top-down participation and emergent rules in marine protected area management in Indonesia. Marine Policy, 34(6):12151225. DOI: $10.1016 /$ j.marpol.2010.04.006

Hanazaki, N.; Tamashiro, J.Y.; Leitão-Filho, H.F.; Begossi, A. (2000) - Diversity of plant uses in two Caiçara communities from Atlantic Forest coast, Brazil. Biodiversity and Conservation, 9(5):597-615. DOI: 10.1023/A:1008920301824

Heck, N.; Dearden, P.; McDonald, A. (2012) - Insights into marine conservation efforts in temperate regions: Marine protected areas on Canada's West Coast. Ocean \& Coastal Management, 57:10-20. DOI: 10.1016/j. ocecoaman.2011.11.008. 
Ivo, C. T. C., Pereira, J. A. (1996) - Sinopse das principais observaçôes sobre as lagostas Panulirus argus (Latreille) e Panulirus laevicauda (Latreille) capturadas em águas costeiras do Brasil, entre os estados do Amapá e do Espírito Santo. Boletim Técnico Cientifico CEPENE (ISSN: 0104-6411), 4(1):7-94, Tamandaré, PE, Brasil. Disponível em http://www4.icmbio.gov.br/cepene/ index.php?id_menu=51\&arquivo=modulos $/$ boletim $/$ res . php\&id_arq=26

Jamieson, G.S.; Levings, C.O. (2001) - Marine protected areas in Canada: implications for both conservation and fisheries management. Canadian Journal of Fisheries and Aquatic Sciences, 58(1):138-156. DOI: 10.1139/f00-233.

Kelleher, G. (1999) - Guidelines for Marine Protected Areas. 107 p., IUCN - International Union for the Conservation of Nature and Natural Resources, Cambridge, U.K. ISBN: 2-8317-0505-3. Disponível em http://data.iucn. org/dbtw-wpd/edocs/PAG-003.pdf.

Kenchington, R. (2010) - Strategic roles of marine protected areas in ecosystem scale conservation. Bulletin of Marine Science (ISSN 0007-4977), 86(2):303-313, University of Miami, Rosenstiel School of Marine and Atmospheric Science, Miami, FL, U.S.A. Disponível em http://www.ingentaconnect.com/content/umrsmas/ bullmar/2010/00000086/00000002/art00011

Marinho, R.A. (2010) - Co-gestão como ferramenta de ordenamento para a pesca de pequena escala do litoral leste do Ceará - Brasil. 225p., Tese de Doutorado, Universidade Federal do Ceará, Fortaleza, CE, Brasil. Disponível em http://www.repositorio.ufc.br:8080/ri/ handle/123456789/1418? mode =full

Menezes, N. A; Figueiredo, J. L. (1985) - Manual de peixes marinhos do sudeste do Brasil: V Teleostei. 154p., Editora da Universidade de São Paulo, São Paulo, SP, Brasil. ISBN: 9000000470026.

Morais, J.O.; Freire, G.S. (2003) - Plataforma ContinentalAs Unidades Geoambientais. Diagnóstico Geoambiental. In: Campos, A. Alves (org.), A Zona Costeira do Ceará: Diagnóstico para a gestão Integrada, pp. 40-41, Aquasis, Fortaleza, CE, Brasil. ISBN: 85-89491-01-3.

Nascimento, R.C. (2006) - Impactos Sócio-Ambientais de Marambaias para a pesca de Lagosta: O Caso de Ponta Grossa, Icapuí-CE. Revista Mercator (ISSN 1984-2201), 5(9):133, Fortaleza, CE, Brasil. Disponível em http:// www.redalyc.org/articulo.oa?id=273620669021

Oliveira, L. (2002) - A percepção da qualidade ambiental. Caderno de Geografia (ISSN: 0103-8427), 12(18):29-42, Minas Gerais, BH, Brasil.

Ransom, K.P.; Mangi, S.C. (2010) - Valuing Recreational Benefits of Coral Reefs: The Case of Mombasa Marine National Park and Reserve, Kenya. Environmental Management, 45(1):145-154. DOI: 10.1007/s00267-0099402-9

Read, A.D.; West, R.J.; Haste, M.; Jordan, A. (2011) Optimizing voluntary compliance in marine protected areas: A comparison of recreational fisher and enforcement officer perspectives using multi-criteria analysis. Journal of Environmental Management, 92(10):2558-2567. DOI: 10.1016/j.jenvman.2011.05.022
Rios, L.V.; Salas,S.; Bello-Pineda, J.; Peniche, I.A. (2007) Distribution patterns of spiny lobster (Panulirus argus) at Alacranes reef, Yucatan: spatial analysis and inference of preferencial habitat. Fisheries Research, 87(1):35-45. DOI: 10.1016/j.fishres.2007.06.021

Salles, R. (2011) - Avaliação econômica e ambiental dos sistemas de pesca utilizados nos municípios de Aracati e Icapui, subsídios para a gestão. 144p., Tese de Doutorado, Universidade Federal do Ceará, Fortaleza, CE, Brasil. Disponível em http://www.repositorio.ufc.br:8080/ri/ bitstream/123456789/1420/1/2011-tese-rsalles.pdf

Scholz, A.; Bonzon, K.; Fujita, R.; Benjamin, N.; Woodling, N.; Black, P.; Steinback, C. (2004) - Participatory socioeconomic analysis: drawing on fishermen's knowledge for marine protected area planning in California. Marine Policy, 28(4):335-349. DOI: 10.1016/j.marpol.2003.09.003

Silva, S.M.M.C.; Rocha, C.A. (1999) - Embarcações, aparelhos e métodos de pesca utilizados nas pescarias de lagosta no Estado do Ceará. Arquivo de Ciências do Mar (ISSN 0374-5686), 32:7-27, Fortaleza, CE, Brasil. Disponível em http://www.labomar.ufc.br/images/stories/arquivos/ ArqCienMar/V32_1999/acm_1999_32_01.pdf

Silvano, R.A.M.; Begossi, A. (2012) - Fishermen's local ecological knowledge on Southeastern Brazilian coastal fishes: contributions to research, conservation, and management. Neotropical Ichthyology, 10(1):133-147. DOI: 10.1590/S1679-62252012000100013.

Suuronen, P.; Jounela, P.; Tschernij, V. (2010) - Fishermen responses on marine protected areas in the Baltic cod fishery. Marine Policy, 34:237-243. DOI: 10.1016/j. marpol.2009.07.001

Trenouth, A.L.; Harte, C.; Heer, C.P.; Dewan, K.; Grage, A.; Primo, C.; Campbell, M.L. (2012) - Public perception of marine and coastal protected areas in Tasmania, Australia: Importance, management and hazards. Ocean \& Coastal Management, 67:19-29. DOI: 10.1016/j. ocecoaman.2012.04.007

Voyer, M.; Gladstone, W.; Goodall, H. (2012) - Methods of social assessment in Marine Protected Area planning: Is public participation enough?. Marine Policy, 36(2):432439. DOI: 10.1016/j.marpol.2011.08.002 


\section{Anexo 1}

QUESTIONÁRIO SÓCIO-AMBIENTAL

\section{Perfil Geral}

Idade $\begin{aligned} & \text { Sexo }(\quad) \text { M } \\ & \text { Origem ( ) Nativo }\end{aligned} \quad(\quad)$ F

Mora há quanto tempo em Picos?

Quantas pessoas moram na mesma casa?

\section{Perfil Sócio-Econômico e Ambiental}

Com o que você trabalha? Com que frequencia? Apenas em Picos?

Como você utiliza a praia de Picos?

O que vocês pescam? Quais outros animais vocês observam na praia de Picos?

Que plantas vocês cultivam? Para que?

Quais animais vocês criam para comer?

Você gosta de morar em Picos? O que tem de bom em Picos?

Picos mudou ao longo dos anos? ( ) Sim ( ) Não; ( ) Melhorou ( ) Piorou

Quais os problemas daqui?

O que você acha do turismo? （ ) Bom （）Ruim （） Tanto faz

\section{Perfil Gestor}

Você gostaria que a praia de Picos fosse uma área ambiental protegida?

Você estaria disposto a colaborar com a gestão (cuidar) da UC ? De que forma? 